\title{
A Path loss Sensitive Stable Routing Protocol for MANET
}

\author{
Sonal Belani \\ M.Tech Scholar CSE Dept. \\ Patel College of Science and \\ Technology, \\ Bhopal, India
}

\author{
Prof. Parmalik Kumar \\ Assistant Professor, CSE Dept. \\ Patel College of Science and \\ Technology, \\ Bhopal, India
}

\author{
Prof. Hitesh Gupta \\ H.O.D., CSE Dept. \\ Patel College of Science and \\ Technology, \\ Bhopal, India
}

\begin{abstract}
A Mobile Ad Hoc Network is a collection of portable devices that establish communication without the help of any infrastructure such as base stations or access points. As the MANET is infrastructure less, it is having dynamic nature of random network topology. Due to movement of nodes, the link between nodes breaks. The network needs to find a new route from source to destination. In order to find a route network initiate a route discovery procedure by broadcasting route request packets. The frequent invention of route increases the routing load and end to end delay. The existing ad hoc routing protocols based on hop count metric which doesn't take in its consideration the stability of the link. The requirement is to discover a routing protocol that considers link stability during discovery of route, so resulting route is more stable than existing routing protocol. In this paper we compare performance of AODV and Path loss sensitive AODV which finds a stable path using received signal strength.
\end{abstract}

\section{General Terms \\ MANET routing}

\section{Keywords}

AODV, Stability, MANET, Routing, Cross-Layer, Path Loss

\section{INTRODUCTION}

A Mobile Ad Hoc Network (also called MANET) is a collection of mobile nodes that establish communication without the help of any infrastructure [1]. Furthermore, Mobile Ad hoc networks are easy to deploy and does not require any back bone support. MANET is Useful in the absence of infrastructure. Mobile Ad Hoc networks should be self-organizing, multi-hopping, mobile and scalable. Each device in a MANET is free to move independently in any direction, therefore change its links to other devices over and over again. Each node in MANET is equipped to continuously maintain the information regarding route. Topology of the adhoc network depends on the transmission power of the nodes and the location of the portable nodes, which may change from time to time [1].

The main goal of Ad Hoc routing is to send data packets among nodes distributed randomly in the network. Since mobile ad hoc networks have random topology, routing in such networks is a tough task. There is so much work has been done on routing in ad hoc networks [1]. The routes are created as and when required in on-demand or reactive routing protocols. Route discovery mechanisms are used to find a path from source to destination. To maintain low control overhead and reduce the network load only the routes that are currently in use are maintained [1].

Existing routing protocols in ad-hoc networks maintains single route per source and destination node pair. Links in a route may become temporarily unavailable due to node mobility, node failures and the dynamic characteristics of the radio channel [1]. If a link in the route is unavailable then local recovery is initiated, which will find an alternative link for the same route. On the other hand if local recovery fails, then route discovery procedure is initiated to find a fresh route. The frequent route discoveries may increase the routing load of the network. To overcome the situation if multiple routes are maintained then it will increase the control overhead of the network. There is a need to find a route which is stable and remain active for a long duration. There can be a less no of route failures if the route is stable. A stable route reduces number of route discoveries and in turn reduces the routing load.

In this paper, a Path loss sensitive stable routing protocol is proposed which is based on Existing AODV protocol. In proposed protocol try has been made to improve existing AODV by providing a stable path, which is formed by considering path loss as a parameter in determining the route. Performances of existing AODV and proposed protocol are also compared.

\section{BACKGROUND}

On-Demand routing protocols finds the route as and when it is required. These protocols are reactive in nature. The proposed protocol is based on AODV (Ad hoc on demand distance vector routing) protocol. So, the discussion is limited to AODV which is follows.

\subsection{Ad-hoc On-Demand Distance Vector Routing (AODV)}

AODV is a reactive protocol that discovers routes as and when needed. It uses routing tables with one entry per destination. RREQ (route request) packet is sent by the sending node whenever there is a need to find a route. The RREQ traverses from intermediate node to its intended recipient. In response to RREQ packet the RREP (route reply) packet is sent by the destination. To prevent routing loops and 
maintain the freshness of route AODV uses sequence numbers, which are maintained at each destination for [6]. All routing packets carry these sequence numbers. Timer-based states are maintained in each node to remove the unused or older entries in routing table. List of precursors is maintained for each routing table entry, indicating the neighboring nodes sets which use that entry to route packets. The nodes in a precursor list are notified by RREQ (route error) packet whenever the next-hop links breaks. These RREQ packets are forwarded by each predecessor node to its Predecessors, effectively erasing all routes currently using the broken link. [6].

The advantage of AODV is that only active routes are maintained, it requires less memory space which in turn increases the performance. The disadvantages of AODV are, it is not scalable, and it does not perform well in large networks and does not support asymmetric links.

\section{RELATED WORK}

\subsection{Stable path with Different Approaches}

There are various parameters by which we can improve the stability of route in existing routing protocols [2]. These parameters are node residual energy [10], link expiration time [11], Stability of nodes [12], probabilistic link lifetime estimation [13], success rate of data transmission for node [14], link usage [15], and received signal strength (path loss).

\subsection{Received signal strength is better parameter for stability.}

The hop count is used to determine the route in existing on demand routing protocols for ad hoc network, which does not take in its consideration the quality of link while deciding the route. The route found using received signal strength can be more stable than the route found using minimum hop basis. Simulation in [3] shows good resemblance with actual implementation.

\subsection{Cross layer design.}

In a cross layer design the parameter measured at physical layer can be made available to layers above it. The value measured at physical layer is passed to MAC layer and network layer. The received signal strength information sent by the physical layer may be stored either in routing table or neighbor table and can be used to make routing decisions. Such a cross layer design has been utilized to improve the performance of mobile ad hoc network [4].

Received signal strength of RREQ packet can be measured at physical level and based on that routing decisions can be made at routing layer [4]. If RREQ packet has a poor received signal strength then receiving node should not forward the RREQ to its neighbors, so weak link does not participate in the route. The received signal strength can be compared with a predetermined threshold to determine whether it is proper or not. The threshold can be a fixed threshold or an adaptive threshold whose value changes with changing speed of the nodes. The adaptive threshold also adapts to moving direction of the nodes.

The path loss of the link can be found by measuring the received signal strength by cross-layer approach [5]. The path loss measured for each link of an entire route can be added to find cumulative path loss of entire route. The average path loss for route can be found by dividing total path loss with hop count. Only the route which is having less path loss can be selected, so resulting route can be more stable.

The AODV-RSS in [6] determines paths that are long lived means, a route that can sustain for a longer time. The longlived path routing algorithm uses the Received Signal Strength RSS and Received Signal Strength changing rate $\Delta$ RSS to predict the link available time (LAT) between two mobile nodes. AODV-RSS can improve the route quality in route connection time, and route reestablishment frequency.

The Route Stability based QOS Routing (RSQR) protocol proposed in [8] which is an extension of QOS routing with throughput and delay constraints. In order to guarantee the suitable data path for adequate longer duration in MANET, an easy model has been proposed for measuring the link stability and route stability depending on received signal strength. Some additional fields in route request/ reply packets is taken into consideration so that the route stability information can be used to choose a route with increased stability when compared to all possible routes among existing source destination pair.

\section{PATH LOSS SENSITIVE STABLE ROUTE}

AODV broadcast RREQ (route request) packet to all neighbors in order to determine the route. The destination address is checked by neighbors and accordingly they forward RREQ to their neighbors. A RREQ packet traversing from different path arrives at the destination. The path with minimum hop is selected as a route [6]. The route found by AODV may have weak links which may lead to frequent route failures [3]. Frequent route failures will increase the number of route discoveries and in turn increase the routing overhead of the network.

The proposed protocol focuses on minimizing the routing overhead of the network. The path loss of the link is determined by measuring received signal strength of RREQ packet using cross layer design and subtracting it from transmit power. A RREQ packet is only forwarded if the link has sufficient path loss. So, the links with high path loss i.e. weak links may not participate in formation of route.

To decide whether the link has the sufficient path loss or not, the path loss of link is checked against a predetermined threshold.

The threshold to be used can be a fixed value or it could be changing adaptively with changing network condition.

As shown in Figure 1 fixed threshold design compares path loss of link with predetermined threshold which is the desired value of the path loss. The RREQ is only forwarded if the path loss of the link is greater than the threshold, so links having high path loss may not participate in formation of route. The route found by fixed threshold method is stable and remains active for longer time. The fixed threshold does not consider the moving direction of sending and receiving node. Reduced route discovery and reduced link failures can be achieved by fixed threshold, but overall performance may not be satisfactory due to increased no of hops in stable route. The increased no of hops increases the end to end delay and reduces the throughput of the network. 


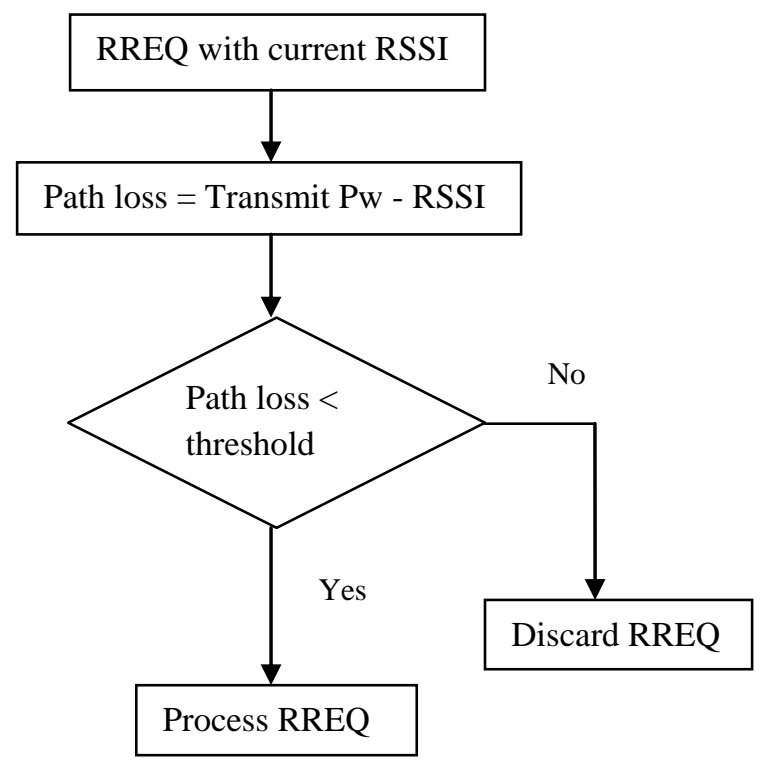

Figure 1: shows flowchart for finding stable path using path loss as a parameter. RREQ with higher path loss than predetermine threshold will be discarded.

To improve the performance the adaptive threshold is used. Which uses different values of threshold with reference to moving speed of the nodes, in addition it also considers moving direction of nodes. Figure 2 shows the path loss of link is stored in the Neighbor table (NT) against the address of the neighboring node from which it is received. So, whenever node receives RREQ from neighbors the current path loss is checked against the previous path loss stored in the table. If the current path loss is lesser than the previous path loss, it indicates that the nodes are approaching towards each other. If the current path loss is greater than previous path loss, it indicates that the nodes are moving away from each other.

Whenever nodes receive RREQ from the neighbors its received signal strength is used to calculate the path loss of the link. The calculated path loss is checked against a threshold. If the path loss is greater than the threshold but the nodes are approaching then nodes will forward the RREQ. If the path loss is greater than the threshold but the nodes are moving away from each other then the nodes discards the RREQ. If the received signal strength is greater than the threshold then the nodes forwards RREQ.

\section{RREQ with current RSS}

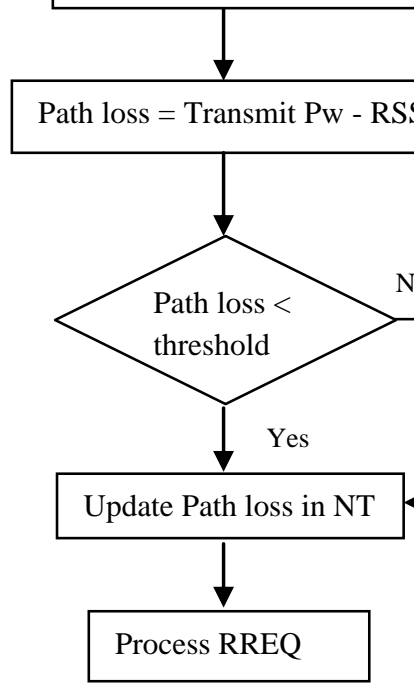

Figure 2: shows flowchart for finding moving direction of mobile nodes using path loss of the link. Mobile nodes are either approaching or moving away from each other so RREQ is processed accordingly.

\section{Simulation Environment and Result Analysis}

There are various simulators available to implement wireless as hoc network. Ns-2.34 simulator is used for testing proposed protocol. Ns-2.34 has support for simulating a multi-hop wireless ad-hoc environment.

Random topology is generated which contains 49 nodes deployed in an area of $1000 \times 1000 \mathrm{~m}^{2}$. Movement generation files are used for different speeds in the range of $5 \mathrm{~m} / \mathrm{s}$ to 25 $\mathrm{m} / \mathrm{s}$. Traffic pattern with 10 different seeds are used to generate traffic. Each point in result is an average of 10 seeds. The other simulation parameters are summarized in "table 1".

Following three cases are tested

(1) AODV

(2) AODV with path loss and Fixed Threshold (AODV Fixpath).

(3) AODV with path loss and adaptive threshold which considers moving direction of nodes (AODV Adpath).

The fixed threshold value of 200 (path loss which is measured after traveling 200 meters) is used for "AODV Fixpath" and it is tested for different speed in the range of $5 \mathrm{~m} / \mathrm{s}-25 \mathrm{~m} / \mathrm{s}$.

The different threshold values $(230 \mathrm{~m}, 210 \mathrm{~m}, 200 \mathrm{~m}, 187.5$ $\mathrm{m}, 162.5 \mathrm{~m})$ are used for "AODV Adpath" for the speeds (5 $\mathrm{m} / \mathrm{s}, 10 \mathrm{~m} / \mathrm{s}, 15 \mathrm{~m} / \mathrm{s}, 20 \mathrm{~m} / \mathrm{s}, 25 \mathrm{~m} / \mathrm{s}$ ) respectively. In addition to that it also considers the moving direction of the mobile nodes.

Table 1 Simulation Parameters

\begin{tabular}{|c|c|}
\hline Parameters & Values \\
\hline Transmission range & 250 meter \\
\hline Simulation time & $200 \mathrm{sec}$ \\
\hline Transmission protocol & TCP \\
\hline Routing protocol & $\begin{array}{c}\text { AODV, AODV Fixpath and } \\
\text { AODV Adpath. }\end{array}$ \\
\hline Packet size & 512 Bytes \\
\hline
\end{tabular}

\section{PACKET DELIVERY RATIO}

The packet delivery ratio can be determined by dividing no of packets received by number of packet sent. Figure 3 shows the packet delivery ratio for AODV, AODV Fixpath and AODV Adpath. Both AODV Fixpath and AODV Adpath improve the packet delivery ratio than AODV.

\section{NORMALIZED ROUTING LOAD}

The normalized routing load is the overhead on the network in order to find and maintain the route. Normalized routing load can be determined by finding number of routing packets sent ner number of data packet received. From Figure 4 it can be Update Path loss in NT erved that AODV has more routing overhead compared to both the AODV Fixed and AODV Adaptive. Both the variants of AODV do not process the RREQ if it is not having Discard RREQ ficient received signal strength that will reduce the routing 
load in both the variants. The AODV does not have stable route which increases the route discovery. Increased route discovery incurs more routing overhead.

\section{AVERAGE END TO END DELAY}

The average end to end delay of the route can be determined by finding the total delay and dividing it by hop count. From Figure 5 we can observe that AODV achieves reduction in average end to end delay. This can happen because AODV has the minimum hop route and both AODV Fixpath and AODV Adpath has a route with higher no of hops than AODV.

\section{THROUGHPUT}

The total data transmitted per second is called throughput of network.

It can be observed form Figure 6 that both AODV Fixpath and AODV Adpath achieve higher throughput than AODV.

AODV has a better end to end delay but it does not have a stable route. The frequent route discovery increases the routing load which reduces the throughput.

\section{FREQUENCY OF ROUTE REQUEST}

The Frequency of route request is no of route request per second.

It can be observed from the Figure 7 that AODV has the higher frequency of route request compared to both AODV Fixpath and AODV Adpath. Due to the stable path found by both AODV Fixpath and AODV Adpath they achieve lesser frequency of route request.

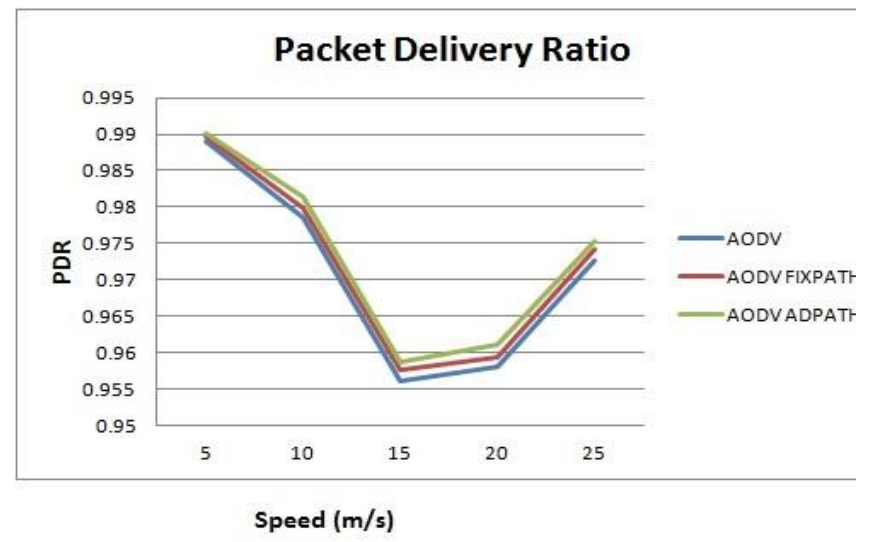

Fig. 3 shows packet delivery ratio against different speeds of mobile nodes. It indicates both the variants of AODV have a good performance in terms of packet delivery ratio.

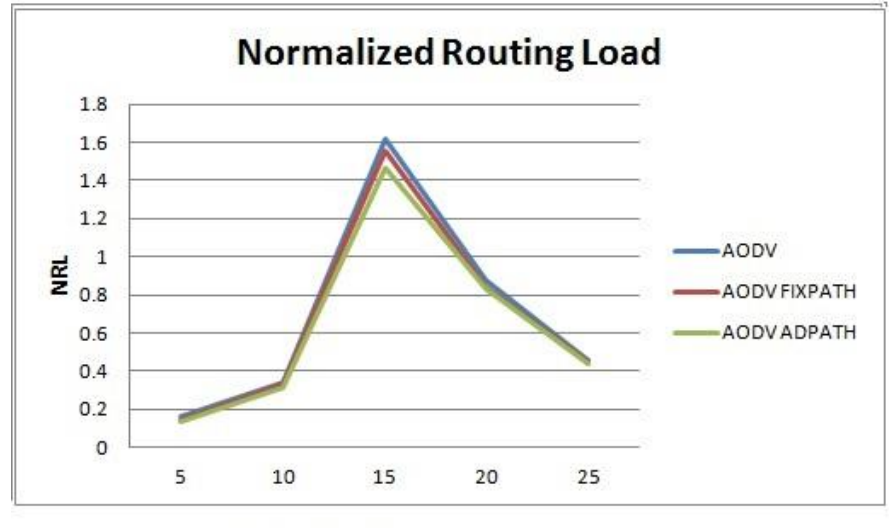

Speed $(\mathrm{m} / \mathrm{s})$

Fig. 4 shows normalized routing load by varying the speeds of mobile nodes. It is indicating that AODV is having higher routing load compared to both the variants of AODV.

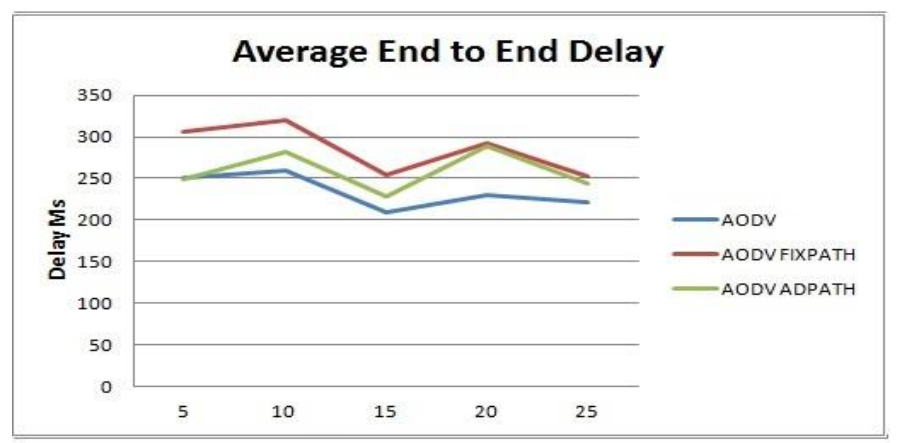

Speed $(\mathrm{m} / \mathrm{s})$

Fig. 5 shows average end to end delay occurred by varying the speed of mobile nodes. Average end to end delay of AODV seems better than both the variants of AODV.

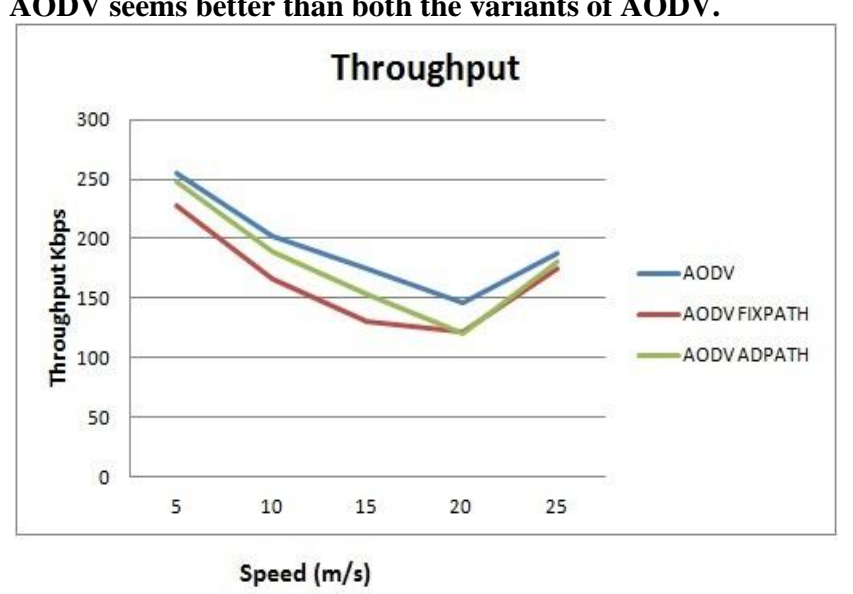

Fig. 6 shows throughput by varying the speed of mobile nodes. Both the variants of AODV seem to achieve better throughput than AODV. 


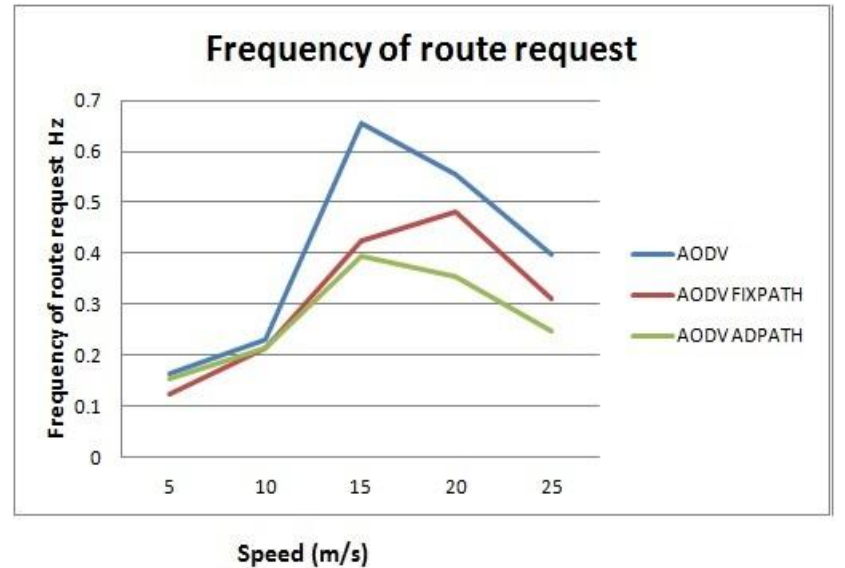

Fig. 7 shows Frequency of route request by varying the speed of mobile nodes. Both the variants of AODV seem to have to have lesser frequency of route request than AODV.

\section{CONCLUSION}

In this paper the performance of AODV and two proposed variation of AODV with added path loss sensitive feature are evaluated. It has been suggested by the results that Both AODV Fixpath and AODV Adpath achieves higher packet delivery ratio and throughput compared to existing AODV protocol. The Stable path found by both the proposed methods reduces frequent route failures and as a result reduces the routing load of the network.

The AODV strives for a minimum hop path which reduces the average end to end delay. On the other hand the stable path is having more no of hops compared to AODV which will increase end to end delay. The stable path obtained by both the proposed method achieves lesser frequency of route request compared to AODV. The stable path found by path loss sensitive approach may increase the end to end delay compared to AODV but it improves the quality of service of the network.

\section{REFERENCES}

[1] H.D.Trung, W.Benjapolakul,P.M.Duc, "Performance evaluation and Comparison of different ad hoc routing protocols", Department of Electrical Engineering, Chulalongkorn University, Bangkok, Thailand, May 2007.

[2] Hadi Sargolzaey, Ayyoub Akbari Moghanjoughi and Sabira Khatun "A Review and Comparison of Reliable Unicast Routing Protocols For Mobile Ad Hoc Networks", International Journal of Computer Science and Network Security (IJCSNS), VOL.9 No.1, January 2009.
[3] Kwan-Wu Chin, John Judge, Aidan Williams and Roger Kermode, "Implementation Experience with MANET Routing Protocols", ACM SIGCOMM Computer Communications Review, Volume 32, Number 5, November 2002.Tavel, P. 2007 Modeling and Simulation Design. AK Peters Ltd.

[4] B.Ramchandran and S. shanmugavel, "Received Signal Strength-based Cross-layer design for Mobile Ad Hoc Networks", IETE Technical Review, Volume 25, Issue 4, JUL-AUG 2008.

[5] Boumedjout Amel, Mekkakia Maaza Zoulikha, "Routing Technique with Cross-layer Approach in Ad Hoc Network", IEEE, 2009

[6] Charles E. Perkins, Elizabeth M. Belding-Royer, Samir R. Das, "Ad hoc On-Demand Distance Vector (AODV) Routing draft-ietf-manet-aodv-13.txt,", Mobile Ad Hoc Networking Working Group, INTERNET DRAFT, 17 February 2003.

[7] Ruay-Shiung Chang and Shing-Jiuan Leu,'Long-lived Path Routing With Received Signal Strength for Ad Hoc Networks", IEEE International Symposium on Wireless Pervasive Computing, January 2006

[8] Prof. Shitalkumar Jain and Miss.Sunita I. Usturge, "Signal Strength Based Congestion Control in In MANET", IISTE,Advances in Physics Theories and Applications, ISSN 2224-719X (Paper), ISSN 2225-0638 (Online), Vol 1, 2011

[9] Nityananda Sarma and Sukumar Nandi, "Route Stability Based QoS Routing in Mobile Adhoc Networks", Wireless Personal Communication, Vol-54, pp-203-224, 2010.

[10] Fang Xie, Lei Du, Yong Bai, Lan Chen, "Energy Aware Reliable Routing Protocol for Mobile Ad-HocNetworks", IEEE Communication Society, WCNC 2007proceedings.

[11] Nen-Chung Wang, Yung-Fa Huang, Jhu-Chan Chen, "A table weight-based on-demand routing protocol for mobile ad hoc networks", Elsevier Information Sciences 177, 2007, p 5522-5537

[12] Athanasios Bamis, Azzedine Boukerche, Ioannis Chatzigiannakis, Sotiris Nikoletseas, "A mobility aware Protocol synthesis for efficient routing in ad hoc mobile Networks", Elsevier Computer Networks 52, 2008 P130 154

[13] Zhao Cheng, Wendi B. Heinzelman, "Discovering long lifetime routes in mobile ad hoc networks", Elsevier Ad Hoc Networks 6, 2008, p661-674.

[14] Imad Jawhar, Zouheir Trabelsi, and Jameela AlJaroodi,"Towards More Reliable Source Routing in Wireless Networks", IEEE

[15] Xie Xiaochuan, Wei Gang, Wu Keping, Wang Gang, Jia Shilou, "Link reliability based hybrid routing for tactical mobile ad hoc network", Elsevier Journal of Systems Engineering and Electronics Vol. 19, No. 2, 2008, pp.259-267. 